\title{
NASA LIVE: Communicating Knowledge to the University Community
}

\author{
Katrina L. Townes-Young \\ NASA LIVE Program Manager \\ NASA Langley Research Center \\ Center for Distance Learning
}

According to NASA Adminstrator, Sean O'Keefe, NASA's mission is "to understand and protect our home planet; to explore the Universe and search for life; and to inspire the next generation of explorers... as only NASA can" (2002). NASA Langley Research Center (LaRC), located in Hampton, Virginia, is one of NASA's leading aeronautical and aerospace research facilities that employs thousands of engineers and scientists who produce cutting edge research and technology. With resident experts, research findings, and commercial applications, Hosted by NASA's Center for Distance Learning, we recognize that access to such individuals and information would offer students and faculty across the nation the unique experience of making real world connections to the science, technology, engineering, and mathematics taught in the classroom, as only NASA can. One method of real-time access that was researched and explored was videoconferencing; videoconferencing "is the transmission of images (video) and (audio) back and forth between two or more physically separate locations" (International Trade Data Network, 1999). Therefore, NASA LIVE (Learning thorugh Interactive Videoconferencing Experiences) represents a new dimension in communicating knowledge and educational outreach to faculty and students in an interactive, virtual setting.

According to Jeffrey Branzburg, "videoconferencing allows you to bring resources into your classroom that you may not be able to experience in "real life" (2001). Debuting in Fall 2001, NASA LIVE is a series of FREE, 60-minute videoconferencing programs for colleges and universities. NASA LIVE is designed to: (1) communicate NASA knowledge to faculty and students in order to increase scientific, technological, engineering, and mathematical literacy; (2) increase faculty and student interest and participation in NASA and related programs; (3) provide faculty and student with a network of NASA mentors and colleagues working on programs, projects, and research in aeronautics, space technology, atmospheric science, and related fields; and (4) support the mission of the various collegiate and professional organizations by establishing a link between NASA and the university community. Each videoconference features a NASA expert who provides an overview of a past, present, or future program or project and a question and answer session. In addition, these videoconferences utilize the latest multimedia animations, video footage, web-based components, and research data. Following each videoconference program, faculty and students are also made aware of NASA LaRC's faculty support program (.i.e, 
NASA Faculty Fellowship Program (NFFP), National Research Council Postdoctoral Program, etc., NASA Educator Workshop (NEW), etc.) and student support programs such as (internships, Langley Research Summer Scholars Program (LaRSS), Graduate Student Research Program (GSRP), co-ops, and other employment opportunities.)

In order to participate in a NASA LIVE event, faculty and students can access a web-based menu of over 20 aeronautics, aerospace, atmospheric sciences and other related topics at http://live.larc.nasa.gov. After selecting a topic or topics of choice, faculty or students have the ability to request a videoconferencing presentation at a date and time which is convenient to the requester. Upon receipt of the request/registration, the faculty or student would be notified and given a confirmation of the presentation. Moreover, the faculty and students also have an opportunity to gain additional information related to the videoconference from the NASA LIVE Web site. The website features a program description, presenter biography, web resources, and other related facts.

NASA LIVE welcomes faculty, professional organizations, and student organizations to participate in the videoconferencing experience. There is not a limit on the number of persons participating in a videoconference. However, it is highly recommended that at least 10 persons be present during the presentation. If there are fewer than 10 persons in an organization, group, class, etc., then they can join with another group through a video bridge. The video bridge allows Multi-Point videoconferences among several (three or more) locations (or sites) with all individuals participating in the same videoconference (like a telephone conference call). An Integrated Services Digital Network (ISDN/H.320) phone line or Internet Protocol (IP/H.323) connection with a PicTel/Tandberg/Vtel type system and a connection speed of 128-786 kilobits per second (kbs) is required to participate in a NASA LIVE_videoconference.

NASA LIVE partners with the American Institute of Aeronautics and Astronautics (AIAA), the world's largest professional society devoted to the progress of engineering and science in aviation, space, and defense. The AIAA continues to be the principal voice, information resource, and publisher for aerospace engineers, scientists, managers, policymakers, students, and educators. In addition, NASA LIVE has an established alliance with the Hispanic Educational Telecommunications Systems (HETS). HETS is a telecommunications consortium of colleges and universities in the United States and Puerto Rico. Its mission is to widen the access of Hispanics to higher education and training opportunities through educational telecommunications and distance learning.

Lastly, we evaluate our program through an online evaluation form at our web site. The evaluator designates a program to evaluate and rates various aspects using a scale of $1=$ Poor $2=$ Fair $3=$ Good $4=$ Excellent, and NA $=$ Not Applicable. They rate Program Format, Usefulness of Program, Program Content, Program Length, Program Facilitator, Presenter(s), Print Resource 
Materials, Multimedia Resources (if available), Audio Quality, Video Quality, Facilitation Set-up, and Facilitation Follow-up. Written comments are solicited on "Did the videoconference (presentation) meet your intended objectives?", "How did this videoconferencing experience compare to a typical instructional experience?", "What would have improved this presentation?", "What should change for future videoconferences?", and "Additional Comments/Suggestions". 